\title{
How service users and carers understand, perceive, rephrase, and communicate about "depressive episode" and "schizophrenia" diagnoses: an international participatory research
}

\author{
Jean-Luc Roelandt ${ }^{1,2,3}$ (D) Antoine Baleige ${ }^{1,2} \cdot$ Marie Koenig ${ }^{1,2} \cdot$ Vincent Demassiet $^{1,2,4} \cdot$ Mohamed Agoub $^{5}$. \\ Victoria Barikova ${ }^{6}$. Dalila Benmessaoud ${ }^{7}$. Floriane Brunet $^{1,2} \cdot$ Mauro-Giovanni Carta $^{8}$. Giulio Castelpietra ${ }^{9}$. \\ David Crepaz-Keay ${ }^{10}$. Nicolas Daumerie ${ }^{1,2}$. Audrey Fontaine ${ }^{1,2}$. Neringa Grigutyte ${ }^{11}$. Jugal Kishore ${ }^{12}$. \\ Marta Kiss $^{13}$. Marc Laporta ${ }^{14}$. Elkhansaa Layoussif ${ }^{5}$. Youssouf Limane ${ }^{15} \cdot$ Marcelino Lopez $^{16}$. Gioia Mura ${ }^{8}$. \\ Jean-François Pelletier ${ }^{17} \cdot$ Mbolatiana Raharinivo $^{18}$. Sami Richa ${ }^{19} \cdot$ Rebecca Robles-Garcia $^{20}$. Anne-Claire Stona ${ }^{1,2}$. \\ Marina Skourteli ${ }^{21}$. Catherine Thévenon ${ }^{1}$. Michel Triantafyllou ${ }^{6}$. Fotis Vasilopoulos ${ }^{21}$. Stéphanie Wooley ${ }^{22}$. \\ Geoffrey Reed $^{23} \cdot$ Mathilde Guernut $^{1,2} \cdot$ Shekhar Saxena ${ }^{23} \cdot$ Françoise Askevis-Leherpeux $^{1,2,3}$
}

Received: 25 September 2019 / Accepted: 3 February 2020 / Published online: 22 February 2020

(c) The Author(s) 2020

\begin{abstract}
Background For ICD-11, the WHO emphasized the clinical utility of communication and the need to involve service users and carers in the revision process.

Aims The objective was to assess whether medical vocabulary was accessible, which kinds of feelings it activated, whether and how users and carers would like to rephrase terms, and whether they used diagnosis to talk about mental health experiences.

Method An innovative protocol focused on two diagnoses (depressive episode and schizophrenia) was implemented in 15 different countries. The same issues were discussed with users and carers: understanding, feelings, rephrasing, and communication.

Results Most participants reported understanding the diagnoses, but associated them with negative feelings. While the negativity of "depressive episode" mostly came from the concept itself, that of "schizophrenia" was largely based on its social impact and stigmatization associated with "mental illness".

When rephrasing "depressive episode", a majority kept the root "depress*", and suppressed the temporal dimension or renamed it. Almost no one suggested a reformulation based on "schizophrenia". Finally, when communicating, no one used the phrase "depressive episode". Some participants used words based on "depress", but no one mentioned "episode". Very few used "schizophrenia".

Conclusion Data revealed a gap between concepts and emotional and cognitive experiences. Both professional and experiential language and knowledge have to be considered as complementary. Consequently, the ICD should be co-constructed by professionals, service users, and carers. It should take the emotional component of language, and the diversity of linguistic and cultural contexts, into account.
\end{abstract}

Keywords International Classification of Diseases $\cdot$ Clinical utility $\cdot$ Communication $\cdot$ Participatory research $\cdot$ Service users $\cdot$ Carers

Jean-Luc Roelandt

jroelandt@epsm-lille-metropole.fr

Extended author information available on the last page of the article

\section{Introduction}

Twenty-seven years after the tenth edition of the International Classification of Diseases (ICD 10, 1992), the 11th version was adopted on May 25, 2019, and will come into effect in January 2022. Future updates should be performed every $1-10$ years. 
While ICD-10 was primarily aimed at professionals, the World Health Organization (WHO) emphasized the clinical utility of the classification as one of the main goals of ICD-11 [1]. As clinical utility is based on communication, the WHO decided for the first time to involve all stakeholders in the revision process, including users of mental health services and carers [2].

This paper illustrates this process in the mental health field.

\section{The case of "mental and behavioral disorders"}

Mental health service users and carers are recognized as "experts by experience" on an international level and this has become one of the guiding principles of mental health policies [3]. As such, participatory research has now become an integral part of the methodology used in health care settings.

Moreover, access to information is considered one of the key dimensions of empowerment for mental health service users and carers [4]. For many years now, providing information has also been recognized as an important therapeutic tool [5]. Finally, it is an ethical and legal prerequisite to the user's decision - through expression of consent-and respect for the person's dignity and autonomy [6]. Taking users' experience of their diagnoses into account should improve the scientific basis and accuracy of diagnoses and communication. As practice and research become more inclusive, professionals need to adopt a language which respects human rights and avoids reductive, labeling, or stigmatizing terms [7]. The changes in naming also reflect changes in medical practice, from an asymmetrical and often paternalistic relationship to a true partnership, as illustrated by the Montreal model of patient partnership [8], and the French citizenship psychiatry movement [9].

Finally, effective communication requires that words and phrasings are accessible and understandable by everyone. Because the ICD is an international standard, and language is not free from culture, the naming of category labels has to be questioned [10]. A direct translation from the English denomination might not be the most appropriate term and phrasing should be discussed with all stakeholders, especially users and carers. Effective communication also entails that the underlying concept exists on both sides, independent of culture or organization of mental health services.

\section{Background}

The French WHO Collaborating Center (WHO-CC, in Lille), in collaboration with the WHO Geneva, and the Medical, Scientific, Health, Mental Health and Society Research Centre (CERMES) organized meetings on the revision process, with the active participation of French speaking professionals and the French Federation of Psychiatry [3]. These meetings of clinicians, researchers, users, and carers led to the creation of a French-speaking consortium to support the WHO in the revision process of the diagnostic classification system.

It was decided that the Canadian WHO-CC (Montreal) would focus on the revision of current Z-coded contextual factors, and the French WHO-CC would focus on F-coded diagnoses and their essential features on an international level.

A first meeting, bringing together all partners, was held prior to the study (Summer 2016), to agree on a consensual international protocol, develop shared materials, train investigators, and discuss translations. A second meeting took place after the end of data collection and analyses (Spring 2018), to discuss and interpret the results, and to agree on recommendations to be addressed to the WHO.

\section{A focus on "depressive episode" and "schizophrenia"}

Even if the diagnoses are different in terms of prevalence, impact, and social representations [11], both are stigmatizing, which can limit access to information and care [12].

"Depression" is now recognized by the WHO as the most common diagnosis of mental disorder and the leading cause of disability worldwide [13]. Although effective treatments exist, fewer than half of those supposedly affected in the world (in some countries, less than 10\%) benefit from those treatments [14], mainly due to a lack of information and negative stereotypes associated with the diagnosis [15]. There is also a lack of consistency in the methods of assessment and the resulting diagnoses, which may have a direct impact on a person's health, either because of the risks associated with unnecessary treatment due to over-diagnosis, or because of the lack of access to care due to under-diagnosis.

"Schizophrenia" is considered the fifth largest disabling diagnosis of mental disorders in the world [13] and the most stigmatizing psychiatric label across countries [16], linked to its historic construction [17]. It is now recognized that carers for people with a diagnosis of "schizophrenia" are also highly impacted, psychologically, socially, and economically [18]. Communication about the diagnosis is a major issue. First, the experience of distress may make it difficult for some users to access information [19]. Second, social stigmatization makes some professionals reluctant to announce this diagnosis [20]. These attitudes contribute to increase the communication gap among and between professionals, users, and carers [21], and to reduce the apparent incidence of "schizophrenia" [22]. To overcome these difficulties, the Japanese Society of Psychiatry and Neurology decided to replace the original term, "Seishin-Burentsu-Byo" (in English: mind-split disease), with a new one, "Togo-ShitchoSho" (in English: integration disorders). This change almost 
doubled the percentage of diagnoses in 3 years (from 36.7 to $69.7 \%$ ) and reduced stigmatization [23]. Some associations and researchers go beyond mere phrasing matters, and argue that "schizophrenia does not exist" and that the concept might be replaced by "psychosis spectrum disorders" [24].

\section{Objectives}

The objectives were to assess whether users and carers:

1. Report understanding the diagnosis of "depressive episode" or "schizophrenia",

2. Associate it with a negative feeling, and, if so, why?

3. Would like to rephrase it, why, and using which terms?

4. Use the diagnosis label when they talk with family and friends about their own (users), or the person they care for (carers), mental health condition.

The first three were extended to essential features of both diagnoses [25].

Results were communicated to the WHO. They were used in making modifications to the final diagnostic guidelines, especially for schizophrenia. These results were used to make the diagnostic guidelines more understandable, less stigmatizing, use more culturally relevant language in descriptions of categories, and to help translations.

\section{Methods}

At the request of the WHO, the French WHO-CC designed an innovative protocol assessing whether ICD vocabulary is accessible, which kinds of feelings it evokes, whether and how users and carers would like to rephrase terms, and whether they use the diagnosis to talk about mental health conditions.

The protocol was implemented from August 2016 to March 2018 in 15 countries (20 research sites): Algeria, Canada, France, Greece, Hungary, India, Italy, Lebanon, Lithuania, Madagascar, Mauritania, Mexico, Morocco, Spain, and UK.

\section{Participation of persons with lived experience}

Users and carers were involved in all the stages of the project [26]: they participated in the development of the protocol, in the review of the design and the materials, in pretesting, and in the interpretation of the results.

\section{Participants and design}

Users were recruited as they visited the mental health services located at the research study sites, with the following inclusion criteria. They needed to have been diagnosed with "depressive episode" or "schizophrenia", and to have attended an outpatient health service.

Carers had to provide assistance to a mental service user, either on a part-time or full-time basis, on a personal basis, and in a continuous manner. As such, professional carers were not included. Carers had to know the diagnosis and were contacted with the consent of the user for whom they were caring. Carers were not necessarily caring for the users who were included in the study ( $43 \%$ for depressive episode, $64 \%$ for schizophrenia), either because users did not have a carer, or because carers did not know the diagnosis or did not consent to participate. Consequently, users' and carers' answers were treated as independent samples.

Interviewers had to be health professionals working in the field of mental health, including peer workers and experts by experience.

\section{Ethical issues}

The protocol complied with the Helsinki Declaration of 1975, as revised in 2008, and the WHO Good Clinical Research Practice (GCRP) guidelines, and each site submitted the protocol for agreement to the local or national relevant authorities. Informed written consent was obtained from every participant.

According to French law, the WHO-CC of Lille received agreement from relevant authorities to upload, store, and analyze databases. The data protection and management procedures were approved by specific regulations in force in France (i.e. the sponsor country).

\section{Procedure}

The study was based on face-to-face questionnaires, focused on either "depressive episode" or "schizophrenia". The questionnaires for users and carers were organized in the same manner, with only some questions specific to one group (users vs. carers). In such cases, users answered for themselves, and carers for the user they cared for.

Questionnaires were composed of two main sections: background information, including socio-demographic data (gender; age; marital status; way of life; family background; occupation; level of education) and a package of four questions about the phrase "depressive episode" or the word "schizophrenia".

1. Understanding Do you understand the phrase (or the word)?

2. Feelings What does this phrase (or this word) bring to mind? [possible answers: something positive-negative or-neither]. For each answer, explain why? 
3. Rephrasing Would you rephrase this phrase (or this word) and why? [possible answers: it is clearer, more understandable - it better matches the current or past lived experience-it is more positive (multiple choices possible)]. If rephrasing, in which term(s)?

4. Communication Can you talk to your family and friends about your (or that of the person you care for) mental health? If yes, what terms do you use?

Each country translated materials from original French and English documents, using a back-forward procedure, and encoded data in one of those two languages.

Overall samples were composed of 279 users and 232 carers for "depressive episode", and of 263 users and 255 carers for "schizophrenia".

\section{Analyses of verbal materials}

- Propositions of rephrasing were classified by two independent coders, with the help of a third one in case of disagreement, into three categories: (1) keeping the etymological roots of the diagnosis: "episod"” and "depress*"for "depressive episode"; "*schizo*" and "*phren*" for "schizophrenia"; (2) using essential features of the diagnosis, either exact or derived words (3) neither type of classification.

- Analysis of terms used to communicate with relatives focused on the first category.

- Motivations of negative feelings were analyzed using the "Iramuteq" quali-quantitative software. Iramuteq, based on hierarchical descending classification [27], allows the extraction of differentiated categories from textual data and the exploration of whether external variables (here: status as user or carer) are associated with them. Categories were labeled as a function of their most representative elements and the context of their occurrence.

\section{Statistical analyses}

Means and standard deviations (SDs) were calculated for continuous variables, and frequency (in percentages) for categorical variables. Comparisons between users and carers were based on Student $t$ or $\chi^{2}$ tests, according to the type of variables contrasted (continuous or categorical, respectively).

Data were analyzed with Statistica version 10.

\section{Results}

Results concerning motivations of feelings, reformulations, and communication are illustrated by quotations of responses from participants worldwide (Appendix 1).

\section{Population}

In line with WHO statistics, a majority of users diagnosed with "depressive episode" were females, and inversely for "schizophrenia" (Table 1).

Regarding the diagnosis of "depressive episode", post hoc analyses showed that users and carers had similar profiles, in terms of age, level of education, and family composition. Nevertheless, compared to carers, users were more often female, single, alone, and without children.

Regarding the diagnosis of "schizophrenia", users and carers were similar in terms of level of education, but differed on other criteria. Compared to users, carers were more often female, older, more often employed and less often single, alone, and childless.

\section{“Depressive episode"}

\section{Understanding}

More than $90 \%$ of users and carers said that they understood the diagnosis label. There was no difference between users and carers (Table 2).

\section{Feelings}

A large majority of users and carers associated "depressive episode" with negative feelings. There was no difference between users and carers.

Analysis of feelings' motivations identified four classes based on 320 (79.6\%) data segments:

1. Class $1(n=185$ [57.8\%]): "negativity of depress*".

2. Class $2(n=52[16.2 \%])$ : associated with carers: "mental illness".

3. Class 3 ( $n=42$ [13.1\%]): "impact on life and difficulties and/or ways to cope".

4. Class $4(n=41$ [12.8\%]): associated with users: "negative thoughts, experience, and memory".

\section{Rephrasing}

About one-third of users and carers suggested a reformulation.

The most frequently quoted reasons for rephrasing were "clearness, understandability" $(n=86$ [38\%]), and "matching experience" ( $n=84$ [37.5\%]) followed by "positivity" $(n=27[12 \%])$.

A significant part of reformulations was based on the "depress" root. A smaller proportion used essential features. Suggestions based on the root "depress*" consisted either in suppressing "episod*" and renaming the diagnosis as "depression" or "depressive state", or in keeping the 
Table 1 Socio-demographic characteristics of service users and carers for the diagnoses of depressive episode and schizophrenia

\begin{tabular}{|c|c|c|c|}
\hline Status & Users & Carers & Statistics \\
\hline Total samples & $n=279$ & $n=232$ & \\
\hline \multicolumn{4}{|l|}{ Depressive episode } \\
\hline Gender: women & $191(68.9 \%)$ & $139(59.9 \%)$ & $\chi^{2}=4.5, d f=1, p=0.03$ \\
\hline Age & $45(14.6,18-84)$ & $44.6(15.1,18-82)$ & $t=0.3, d f=507, p=0.78$ \\
\hline Marital status: single & $91(32.6 \%)$ & $56(24.2 \%)$ & $\chi^{2}=4.3, d f=1, p=0.04$ \\
\hline Way of life: alone & $53(19.1 \%)$ & $19(8.2 \%)$ & $\chi^{2}=12.3, d f=1, p<0001$ \\
\hline Family: no child & $101(36.6 \%)$ & $71(30.7 \%)$ & $\chi^{2}=1.9, d f=1, p=0.16$ \\
\hline Occupation: yes & $122(45.2 \%)$ & $130(56.5 \%)$ & $\chi^{2}=7.6, d f=1, p=0.005$ \\
\hline Age at the end of studies ${ }^{a}$ & $\begin{array}{l}n=244 \\
21.2(6.5,8-52)\end{array}$ & $\begin{array}{l}n=207 \\
21(6.7,9-61)\end{array}$ & $t=0.3, d f=449, p=0.79$ \\
\hline Total samples & $\begin{array}{l}\text { Users } \\
n=263\end{array}$ & $\begin{array}{l}\text { Carers } \\
n=255\end{array}$ & Statistics \\
\hline \multicolumn{4}{|l|}{ Schizophrenia } \\
\hline Gender: women & $84(32.1 \%)$ & $165(64.7 \%)$ & $\chi^{2}=55.2, d f=1, p<0.001$ \\
\hline Age & $39.4(12.1,18-77)$ & $55.1(13.8,18-87)$ & $t=13.8, d f=516, p<0.001$ \\
\hline Marital status: single & $181(68.8 \%)$ & $28(11 \%)$ & $\chi^{2}=180, d f=1, p<0.001$ \\
\hline Way of life: alone & $44(16.7 \%)$ & $18(7.1 \%)$ & $\chi^{2}=11.4, d f=1, p<0.001$ \\
\hline Family: no child & $189(72 \%)$ & $31(12.2 \%)$ & $\chi^{2}=1192, d f=1, p<0.001$ \\
\hline Occupation: yes & $81(31 \%)$ & $110(43.7 \%)$ & $\chi^{2}=8.7, d f=1, p=0.003$ \\
\hline Age at the end of studies ${ }^{\mathrm{a}}$ & $\begin{array}{l}n=243 \\
20.7(5.72,6-50)\end{array}$ & $\begin{array}{l}n=222 \\
21(7.2,10-69)\end{array}$ & $t=0.5, d f=463, p=0.63$ \\
\hline
\end{tabular}

Data are $n(\%)$ or $m(\mathrm{SD})$, range

${ }^{a}$ Based on those declaring to have been schooled

Table 2 Depressive episode: understanding, negative feelings, rephrasing, and talking, as a function of status (service user vs. carer)

\begin{tabular}{llll}
\hline Total samples & Users & Carers & Statistics \\
& 279 & 232 & \\
\hline Understanding, yes & $257(92.4 \%)$ & $208(90.8 \%)$ & $\chi^{2}=0.43, d f=1, p=0.41$ \\
Negative feelings, yes & $237(85.7 \%)$ & $192(83.8 \%)$ & $\chi^{2}=0.40, d f=1, p=0.53$ \\
Rephrasing, yes & $100(36 \%)$ & $76(32.8 \%)$ & $\chi^{2}=0.58, d f=1, p=0.45$ \\
If yes, suggested reformulations" & & & \\
Etymological roots & $51(46.3 \%)$ & $47(56 \%)$ & $\chi^{2}=1.71, d f=1, p=0.19$ \\
"Episod" & $3(2.7 \%)$ & $1(1.2 \%)$ & \\
"Depress" & $45(40.9 \%)$ & $45(53.6 \%)$ & \\
"Episod" and "depress" & $3(2.7 \%)$ & $1(1.2 \%)$ & \\
Other & $59(53.6 \%)$ & $37(44 \%)$ & \\
Essential features & $13(11.8 \%)$ & $6(7.1 \%)$ & \\
Neither & $46(41.8 \%)$ & $31(36.9 \%)$ & \\
Talking, yes & $201(72.6 \%)$ & $178(78.4 \%)$ & \\
If yes, used terms ${ }^{\mathrm{a}}$ & & & \\
Diagnosis & $0(0 \%)$ & $0(0 \%)$ & \\
Etymological roots & $31(16 \%)$ & $60(35.3 \%)$ & \\
"Episod" & $1(0.5 \%)$ & 0 & \\
"Depress" & $30(15.5 \%)$ & $60(35.3 \%)$ & \\
Neither & $162(83.9 \%)$ & $110(64.7 \%)$ & \\
\hline
\end{tabular}

Data are $n(\%)$

${ }^{a}$ All those who wanted rephrasing did not propose a precise reformulation and other ones proposed several possibilities; all those who reported that they could talk with relatives did not specify used terms 
temporal dimension using "period", "phase", or "crisis" instead of "episod*". The most frequently quoted term from essential features was "hopelessness".

\section{Communication}

A similar majority of users and carers said that they could talk to family and friends about their (or the person they care for) mental health condition. There was no difference between users and carers.

Among those who reported communicating with their relatives, no one used the phrase "depressive episode".

\section{"Schizophrenia"}

\section{Understanding}

A majority of users and carers reported understanding the diagnosis. This majority was higher for carers than for users (Table 3).

\section{Feelings}

More than $75 \%$ of users and carers associated "schizophrenia" with negative feelings. There was no difference between users and carers.
Analysis of negative feelings' motivations identified four classes based on $270(77.1 \%)$ data segments:

1. Class 1 (42.2\%): "social and cultural negative side effects of the diagnosis".

2. Class 2 (40\%): "mental illness", sometimes associated with "chronicity".

3. Class 3 (9.3\%): "feeling of fear regarding the word and its image".

4. Class 4 (8.5\%): "difficulties to understand and/or handle the diagnosis".

\section{Rephrasing}

More than one-third of both users and carers suggested a reformulation. Reformulations were most often based on "clearness, understandability" $(n=113$ [38.8\%]), and "matching experience" $(n=82$ [28.2\%), followed by positivity $(n=53[18.2 \%])$. The pattern was the same for users and carers. Almost no one kept the etymological roots of the diagnosis. A small proportion used essential features, most frequently related to modifications of thinking and behavior.

\section{Communication}

A majority, higher for carers than for users, said they can talk to family and friends about their (or the person they
Table 3 Schizophrenia: understanding, negative feelings, rephrasing, and talking, as a function of status (service user vs. carer)

\begin{tabular}{llll}
\hline Total samples & Users & Carers & Statistics \\
& 263 & 255 & \\
\hline Understanding, yes & $168(64.6 \%)$ & $191(76.4 \%)$ & $\chi^{2}=8.49, d f=1, p=0.004$ \\
Negative feelings, yes & $192(75.3 \%)$ & $197(79.4 \%)$ & $\chi^{2}=2.43, d f=1, p=0.12$ \\
Rephrasing, yes & $106(40.3 \%)$ & $91(35.7 \%)$ & $\chi^{2}=1.17, d f=1, p=0.28$ \\
If yes, suggested reformulations" & & & \\
Etymological roots & $5(4.2 \%)$ & $2(2.3 \%)$ & \\
"Schizo" & $2(1.7 \%)$ & 0 & \\
"Phrenia" & $2(1.7 \%)$ & 0 & \\
"Schizo" and "Phrenia" & $1(0.8 \%)$ & $2(2.3 \%)$ & \\
Other & $113(95.8 \%)$ & $87(97.7 \%)$ & \\
Essential features & $17(14.4 \%)$ & $6(6.7 \%)$ & \\
Neither & $96(81.4 \%)$ & $81(91 \%)$ & \\
Talking, yes & $170(65.6 \%)$ & $193(77.2 \%)$ & $\chi^{2}=8.31, d f=1, p=0.004$ \\
If yes, used terms & & & \\
Diagnosis & $28(17.2 \%)$ & $19(10.4 \%)$ & \\
Etymological roots & $1(0.6 \%)$ & 0 & \\
"Schizo" & $1(0.6 \%)$ & 0 & \\
"Phrenia" & 0 & 0 & \\
Neither & $134(82.2 \%)$ & $125(89.6 \%)$ & \\
\hline
\end{tabular}

Data are $n ; \%$

${ }^{a}$ All those who wanted rephrasing did not propose a precise reformulation and other ones proposed several possibilities; all those who reported that they could talk with relatives did not specify used terms 
care for) mental health state condition. Carers communicated more frequently than users.

Among those who reported communicating with their relatives, only a minority of users used the term "schizophrenia".

\section{Discussion}

This project was based on the argument that maximizing clinical utility of ICD-11 would require the strengthening of communication between all stakeholders, and making words and phrasings accessible to everyone.

In line with this argument, an international participatory study conducted in 15 different countries and focused on "depressive episode" and "schizophrenia", examined whether service users and carers reported understanding the diagnosis, whether they associated it with a negative feeling, whether they would prefer rephrasing it, and in which terms, and whether they used the label when talking with family and friends.

Results first confirmed the feasibility and the informativeness of the procedure. They revealed a gap between official medical language and users' and carers' language.

The key conclusion is that the answers of users and carers converge toward similar patterns and conclusions for both diagnoses. A majority reported understanding diagnoses and associated them with negative feelings, and about one-third proposed to rephrase them. Moreover, the fact that users and carers had an overall similar level of education suggests that disagreements with current medical language as used in ICD cannot be attributed to any mental or behavioral disorder.

However, results also revealed specific points of interest for both diagnoses.

Most people reported understanding the phrase "depressive episode", but did not use it to communicate. When communicating and rephrasing, they often kept "depress*", but almost never used "episode". This may suggest that the word "episode" is rarely used when talking about depression. The decision to use "depress*" could be read through the lens of social acceptability, as far as people can identify with depression and think it is possible to recover from it [28]. Nevertheless, the diagnosis, and mostly the word "depression" itself, was associated with negative feelings, showing a match between signifier and signified. Moreover, the fact that the negativity of "depressive episode" is partially based on its status as a mental illness questions its stigmatizing potential due specifically to word choice.

In comparison with "depressive episode", the diagnosis of "schizophrenia" was less accessible, still highly associated with negative feelings, and nevertheless used by some people to communicate. Only a minority of reformulations were based on etymological roots. Linguistic analyses showed that the negativity of the term "schizophrenia" came from two factors: its association with mental illness, and social negative side effects, including stigmatization. Given the evidence that these factors are strongly tied with mental illness [28], this points to a strong conceptual connection between schizophrenia and mental illness, which might explain its low use in rephrasing. Indeed, the medical definition of the disorder differs from its etymological meaning of "mind split", showing that the signifier doesn't match the signified condition.

\section{Limitations}

This study presents some shortcomings, related to the design itself, but also due to the construction and the international character of the ICD.

First, in line with inclusion criteria, all users had their diagnosis disclosed. It implies that professionals communicated at least that one impactful piece of information. Knowing that not all people are given their diagnosis, results and their implications may not be applicable to persons not informed of their diagnosis or outside of the health care system. Moreover, the procedure sometimes raised ethical questions, especially in sites where disclosure of diagnosis was uncommon.

The second limitation is related to the fact that, when the protocol was implemented, the ICD-11 draft was only available in English, with its French translation still ongoing. Moreover, even though the official version of ICD-10 was available in six languages (Arabic, Chinese, English, French, Russian, Spanish), and was translated by national instances in another 36 non-official languages, there was no translation available in two participating sites (India and Madagascar). Finally, translating a word implies that the underlying concept exists, refers to the same elements, and is well rooted in society. It was not the case for all countries involved in the project, suggesting further analyses of their linguistic and cultural specificities. But, even if mentioned as a limitation, it supports the WHO's decision to develop ICD-11 in more languages than its official ones, and to take cultural variations into consideration [10]. This limitation aligns with those inherent in the ICD itself and the difficulty to represent, in an international context, complex concepts with mutually shared terms.

Despite these limitations, results, including examination of verbal answers, suggest a gap between the concepts underlying diagnostics, and emotional and cognitive experiences. Medical language was not always in line with experiential knowledge and often had negative connotations, which could reduce the quality of communication. Moreover, data regarding both diagnoses suggest that rephrasing is mostly based on social and personal acceptability, and alignment with lived experience which may be incorrectly captured 
by medical language. This is in line with what has been described and recognized as experiential knowledge [29], and could be a source of relevant information to reduce the gap between chosen terms and underlying concepts. This would contribute to solving actual issues regarding the diagnosis of "schizophrenia" [21, 24], and potential issues for the diagnosis of "depressive episode".

Some scholars consider medical and experiential knowledge to be related to different semantic and cultural spaces. For them, making a medical classification both accessible and minimally negative and stigmatizing entails adding to ICD-11 a lay version of diagnostic information [30]. Contrary to this point of view, our position is to consider both languages as complementary. Consequently, because communication has to be based on a shared language, we support that a medical classification has to be co-constructed by all stakeholders and take diversity of linguistic and cultural contexts into account. This approach should be in line with the transformation of mental health services to a global approach, consistent with human rights and particularly with the United Nations Convention on the Rights of People with Disabilities.

Acknowledgements The authors are grateful to users and carers who agreed to participate in the study and to Brian Hofer for proofreading.

They also would like to thank the Steering Committee members (Benradia, I., Soussan F., Bousser A., Bretel, J., Brun, P., Julien, J., Larchanche, S., Letailleur, C., Lovell, A., Loubier, D., Marchal, C., Marsili, M., Sebanne, D., Staedel, B., Vasseur-Bacle, S. \& Van Remoortel, J.) and the following colleagues who participated in protocol implementation, data collection, and encoding:

Algeria: Belkacem- Djeffel, M., Boukhatem, I., Boutaleb, D., Mertani, L., Souhlal, Z.

Canada: Morin, F.

France: Alexopoulos, C., Beye, A., Brun, P. Caloone, C., Defromont, L., Demazière, G, Depoorter, C., Desruelle, C., Dumesnil, C., De Fos, E., Lebouteiller, V., Lemarie, N., Mastrangelo, C., Monnier, D., Piquet, T., Saraiva, G., Schmidt, B., Storm, S., Vermeersch, V. Greece: Iatraki, G., Katimertzi, M.

Hungary: Barkász, H., Karácsonyi, A, Meixne, J., Menkó, G., Nyulászi, A., Pálmai, A.

India: Kukreti, P., Rastogi, R, Rathore, K.S., Salodja, U.P., Sethi, S., Singh, S.K., Sood, A., A. Tiwari, Yadav, A.

Italy: Celona, D., d'Oca, S., Farci, V., Garino, D., Marenda, F., Tassi, F., Vitucci, C.M.

Lebanon: Abisaleh, R., Chammai, R. Choueifati, D., Yara, C., Zarzour, $\mathrm{M}$.

Lithunia: Petraškaitė, K., Jurkštaitė-Pačèsienė, L.
Madagascar: Fanomezantsoa, A., Manambisoa, V., Manovosoa, R., Milasoa, L.R., Rabenarivo T. Rahanitrandrasana O., Ramarozatovo, R.V. Razanakolona, Rambolamamy, A., Randriamahefa, M.L., Randriamanantena, M., Rasoamanarivo, T., Ratobimanankasina H.H., Rehedinosy, P.

Mauritania: Abeidak, M., Birame, A. El Ghadi, M., Lemine, M., Mahmoud, M., Sid'Ahmed, M., Yahouvdhou, E.

Mexico: Domínguez, T., Fresán, A., González, A., Hernández, O., Madrigal, P.S., Martínez, N., Medina-Mora, M.E., Muñoz, C., Rascón, M.L., Real, T., Vázquez, A.

Morocco: Abouelfaraj, H., Aitelhaj, M., Cherif Mghari, D., Deroui, K., Fadili, A., Falah, K., Malki, Z., Rachidi, S., Reda, A., Sentoufi, Z. Spain: García, A.R., Iglesias, S.D., Rodriguez, G.B., Colvee, C.B., Luque, L.A., Montero, J.M.T., Morejon, A.V., Pérez, D.M.

Morocco: Abouelfaraj, H., Aitelhaj, M., Cherif Mghari, D., Deroui, K., Fadili, A., Falah, K., Malki, Z., Rachidi, S., Reda, A., Sentoufi, Z.

Funding The study received financial support from the French Ministry of Health for all the necessary on-site travels and international coordination, and the participation of the WHO's Offices in Madagascar and in Mauritania.

Data availability Data are available upon reasonable request to the EPSM Lille-Métropole.

\section{Compliance with ethical standards}

Conflict of interest The authors declare that there are no conflicts of interest.

Open Access This article is licensed under a Creative Commons Attribution 4.0 International License, which permits use, sharing, adaptation, distribution and reproduction in any medium or format, as long as you give appropriate credit to the original author(s) and the source, provide a link to the Creative Commons licence, and indicate if changes were made. The images or other third party material in this article are included in the article's Creative Commons licence, unless indicated otherwise in a credit line to the material. If material is not included in the article's Creative Commons licence and your intended use is not permitted by statutory regulation or exceeds the permitted use, you will need to obtain permission directly from the copyright holder. To view a copy of this licence, visit http://creativecommons.org/licenses/by/4.0/.

\section{Appendix 1}

Quotations from verbatim for depressive episode and schizophrenia: motivations of negative feelings, rephrasing, and communication. 


\section{Depressive episode}

\section{Negative feelings}

Class 1: "negativity of depress"” Users

Depressive makes me think of something negative, Lebanon Depression itself is frightening, France

Depression is always something negative, even if it is an episode rather than a status, but it is negative, Italy

Both depression and episode are negative, $U K$

Carers

It includes the word depressive, obviously it's negative, Lebanon

Because Depression is negative, Lebanon, Mexico

\section{Class 2: "mental illness*"}

\section{Users}

It's a mental illness, Madagascar, Mauritania

Every mental illness is bad, Madagascar

Serious illness that can lead to extreme despair and suicide,

Madagascar

\section{Carers}

Because this term is strong, serious illness, Algeria

It is the disease that is chronic, cannot heal permanently,

Mauritania

Because it's chronic, Morocco

\section{Class 3: "impact on life, and difficulties and/or ways to cope"} Users

Desperate all the time, we don't dare to face life, Madagascar It's not something that will let you handle life in a good way, Italy I am being laughed at by those around me and the community, hopeless for my family and for myself, Madagascar

Because it affects every area of my life, $U K$

\section{Carers}

Because it's the equivalent of being outside of society, Algeria It is very difficult for the person and for others, Spain

Like a refusal, rejection of life and constant despair, Greece

\section{Class 4: "negative thoughts, experience and memory" Users}

When you are depressed, you are not happy and you see all in black, France

(...) all you can feel is sadness and despair, everything you feel is negative, Greece

In relation with lived experience, Algeria

Fear, coldness, unpleasant (...), Lithuania

I immediately think about myself lying in bed, desperate, afraid,

sweaty, with headache and thoughts about death, Mexico

\section{Carers}

I live it and I see it every day and I see that it is negative, France It reminds you of negative memories, France

Painful experience lived, Algeria

Because depression is difficult, unpleasant, tiring, painful thing, Lithuania

\section{Others}

Users

I don't wish that on anyone, you're not yourself, you never find yourself, Spain

Because being depressed means that there is despair, which is not accepted by our religion, Morocco

The absolute sinking, I am trying to see a beam of light within the darkness, Greece

Since it is a diagnosis, it gives me a negative feeling..., Hungary Because it means that the person is down, Lebanon

\section{Temporal dimension}

Users

Depressive crisis/crisis of depression, Italy, Greece

Dark period, Italy

Moment of exhaustion, France

\section{Carers}

Depressive phase, Hungary

Depressive period, Lithuania

Depressive crisis, Lithuania

\section{Essential features}

Users

Hopelessness, Madagascar

Mental fatigue, Madagascar

Sadness, Italy

Lack of interest in life, Mexico

Sadness crisis, Morocco

\section{Carers}

Mental fatigue, Madagascar

Sad moment, Lebanon

Depressive mood, Greece

Moment of sadness, Italy

Others

Users

Madness, Morocco

Anxious period, Hungary

Episode of clinical depression. Difficult to come up with new term

but this term has been over-used and meaning has evolved away

from clinical use, $U K$

A negative mental moment, Algeria

A moment of exhaustion, France

I'm 'out of order', France

Neuropsychological disturbance, Algeria

\section{Carers}

Falling into a psychological hole, France

Unable to see further, to be in a black hole, Spain

The black cloud, Morocco

Episode of need for help, Algeria

Nerve crisis, Morocco

Hidden madness, Mauritania

\section{Communication}

\section{Depress*}

Users

Depression, Greece, India, Italy, Mexico, UK, Algeria, Spain,

France, Lebanon

Depressed, Italy, Mexico, UK

$$
\text { Carers }
$$

Depression, France, Lebanon, Algeria, Canada, Spain, India,

Greece, Italy, Lithuania, Madagascar

Depressed, Mexico, India, France, Italy, Lithuania, Greece

\section{Others}

Users

I don't feel well, Algeria

Fatigue, disgust, difficulty in being optimistic, Canada

In a bad mood, isolation, Spain

I say I burned out to talk about my depression. My body was out of energy. It was due to an accumulation of family and professional problems, France

To my family and friends I say I am not well and to doctors I say I am in a state of depression. I'm afraid that if I use the word depression at work, people will think I'm crazy, France I went crazy, Madagascar 
Carers

Can lead to suicide, Madagascar

...Unpredictability..., UK

\section{Rephrasing}

Depression / depressive state

Users

Depression, Spain, France, India, Italy, Lithuania

Depressive state, Spain

Carers

Depressive symptom, Italy

Depression, Italy, Greece, France, Mexico, Algeria, Lebanon
My head is tired, Madagascar

Hallucinations, obsessions, lack of comfort and fear, Mauritania

I have a form of depression with negative impacts. I talk about it in an open manner, Greece

It has no label, I tell stories. I don't want to, I have no strength,

Hungary

I feel sad, don't feel like doing anything, India

I feel bad, or I have anxiety, or I am sad, or I don't feel well, Italy

I am depressed, diagnosed with post-traumatic depression, in

treatment. I fight, yes, fight is the right word!, Italy

Anxiety, sleep disorders, Lebanon

Depression, tiredness, sadness, hopelessness, Mexico

Hopelessness, like something bad will happen, tearfulness,

desperation, frustration, $U K$

\section{Carers}

Stress, panic attack (...), Greece

Feeling sad, depressed, can't sleep, India

Malaise, difficulty, Italy

Sadness grief guilt, Lebanon

\section{Schizophrenia}

\section{Negative feelings}

Class 1: "social and cultural negative side-effects of the diagnosis"

Users

The word is tied to negative social stereotypes, Hungary It is a socially negative term, Italy

It depresses me, society considers schizophrenic people not normal and stupid, they point fingers at them, exclude them, Hungary

This term has been stigmatized in the media and it affects all schizophrenics (...), France

\section{Carers}

It carries a lot of stigma particularly the social aspect of the stigma. It carries a lot of negative social connotations, people use it when they want to offend somebody, Greece

(...) It is a horrible illness for how it is experienced. Saying you're schizophrenic is worse than saying you have a cancer today, Italy

Because of the stigma, people misinterpret it and think of it as split mind, being aggressive or even criminal. It gives the feeling of total social exclusion, Hungary

Too stigmatizing, Italy

When we say schizophrenic, people immediately think of mentally ill people who will kill everyone (...), France It implies something really negative such as a murderer, a heartless butcher and generally the worst possible associations, Greece

The person who is designed like this is a murderer, belongs to the hospital and should never be let out again. This is the way society thinks. It is not the way I think anymore, Hungary

\section{Class 2: "mental illness", sometimes associated with "chronicity"$$
\text { Users }
$$

It's a mental illness, Algeria, Madagascar, Mauritania, Mexico, Spain

Chronic brain disease, Morocco

Schizophrenia is bad, it ruins your life, it's a horrible disease, makes you feel absent-minded, Mexico

It's an incurable disease, Mauritania

Because it is the illness, each illness is negative experience, Lithuania

Schizophrenia is being sick, not having a good health and not

\section{Rephrasing}

*schizo* or *phrenia*

Users

Frenopatia, Spain

Carers

It is also important to level different types of schizophrenia because not all are the same (...) it can be severe or can be mild, $U K$

\section{Essential features}

Users

Fear of thinking, negative responses to the nervous system and collaboration of thinking, Canada

\section{Carers}

Psychic disease of disorganized thinking, France tress, panic attack (...), Greece

\section{Others}

\section{Users}

Integration disorder, Italy

Hearing voices, Mexico

Disturbance of the mind, disturbance of thinking, thoughts, a

different view of the world, Lithuania

Problems in socialization, behavior and ways of thinking, Greece

Unsound mind, India

Temporarily changed state caused by problems, Hungary

I would call it something else in order to avoid stigma, Greece

Curse of the mind, Madagascar

To be crazy, Morocco

By a more positive term to change the image, Canada

Being taken hostage, Madagascar

Psychic dispute, Lebanon

Anxiety, Spain

Depression, Italy

I would remove the word schizophrenia and specify each symptom, each category, Canada

Loss of the usual prescription of the world, France

Imagination and hearing voices, Algeria

\section{Carers}

Special, different person, Mexico

Mixed emotional color scale, Hungary

Temporarily changed state of mind, Hungary

I don't have a specific idea, but the rephrasing of the disease would be necessary and the application of a more serious media ethics, 
having a good health is a negative thing, Canada

\section{Carers}

A chronic disease, Lebanon

It is a sickness, difficult for the person who lives with it and for the people around, Mexico

Because it is severe incurable illness, Lithuania

Terrible mental illness, Lithuania

It's a dangerous disease, Mauritania

Because it is the most dangerous disease, Morocco

Class 3: "feeling of fear regarding the word and its image" Users

This old Greek word is scary, Hungary

It is often pictured as very scary, dreadful, and connected with aggression. It reminds me of such things too, that is why I don't like to use it for myself, Hungary

It's something bad, I'm scared of the word, Mexico

\section{Carers}

It scares and terrifies me, people use this phrase in a pejorative way. It reminds everyone of a raging madman, a fool. It is offending, scary, it should be modified, Hungary

It's a scary word, it refers to madness, real madness. I still see the reaction of people's fear when I say my son is schizophrenic. It's a label, France

A frightening term, France

Class 4: "difficulties to understand and/or handle the diagnosis"

Users

It hurts, it's difficult to understand, France

This illness is difficult to handle, Algeria

The name schizophrenia doesn't ring a bell but it's a very bad disease, which never heals and is for life, Spain

Not an easy word to say or write. Not understandable, I've already tried to understand but I don't, France

Because I don't understand what it means, Lebanon

Schizophrenia is nothing more than a name that encompasses many diseases, when people say schizophrenia, they don't really know what it is, Mexico

\section{Carers}

Depressive phase,

It is a difficult disease to manage, Algeria

It is a form of mental disorder that at the onset is very difficult to understand (...), Greece

Hurts the people around, Madagascar

\section{Others}

\section{Users}

A view that it is a terrible thing painted by the social world (...), Lithuania

That I'm crazy, Mexico

If you have schizophrenia and you commit a murder, they will take you to the psychiatric hospital not to jail. You have to do something to have schizophrenia., Greece

Makes you lose weight and wealth, Madagascar

Because nothing we do is well done, we are unbalanced, uncontrolled, Spain

Taboo, Morocco

Because all symptoms are negative, Lebanon

Because of behavioral disorders, Algeria

Because the term schizophrenia means disorder of thoughts and ideas, Lebanon

\section{Carers}

When somebody suffers from schizophrenia he suffers in a lot of distress. But when somebody is stable with medication it is not acceptable to use this diagnosis, Greece

It is something psychopathic. Psychopathy, Greece

It's a lot of suffering, Mexico

It's madness, it's something very painful, Mexico

An expression that evokes the division between the physical and the spiritual, Mauritania

It's something that's directly related to psychiatry, Algeria
Hungary

I would use Jim van Os's expression Psychosis Sensibility

Syndrome (PSS), see also Jim van Os Schizophrenia does not exist,

Hungary

Hallucinations, wandering mind, Italy

A deficiency in the brain, Mexico

Non completion of the person's personality, Greece

To lose control on the mind, India

A situation resulting from exposure to extreme stress, Greece

A more detailed expression would be required which also refers to

the seriousness, Hungary

Something more specific to the symptomatology, Greece

Brain disease I don't know more precisely, Canada

Anxiety, Algeria

Headache, Spain

Perturbation of the mind or balance of the mind is better than the word perturbation. There are psychics who have been diagnosed as schizophrenic although they are not crazy, France

I would rename to modify the associated image but I don't know

how, Canada

No idea but important to reformulate, France

Disease that hinders human rights, Madagascar

To be possessed, Morocco

\section{Communication}

*schizo*

Users

I say I suffer from schizophrenic disorders, France

Carers

I tell them that she has schizophrenia, can hear voices, India

Others

Users

Obsessive compulsive disorder with memory problems and isolation, Italy

Language, simple language, anxiety, insomnia, no appetite, lay in

bed all the time, fear is present, Lithuania

Hearing voices, inner debate, problem need of change of

perspective, inner uncertainty, tension Hungary

Depression, sadness, anger, loneliness, Mexico

In terms of feeling well or unwell or that I am feeling bad, Greece

Everyone wants to kill me and is attacking me, India

Sometimes I use the word illness, but mostly the words state or

worry, anxiety, hearing voices, Hungary

I'm sick, I have a mental illness, Mexico

People say I'm crazy, Madagascar

I am talking about severe depression but not schizophrenia, France

No specific words, we talk about it in general, Morocco

That I have paranoias, Spain

Prayer for recovery, Mauritania

\section{Carers}

Locked at home, self-arrested, Italy

Panic attack, anxiety, Lithuania

Mental illness, depression, Greece

Recovery, state, changing states, Hungary

Mentally ill, Mexico

Do not use word schizophrenia, say what to do, how to help and what could help, Lithuania

Simple, natural words mood deterioration, tension, Lithuania

Disease, episode, swing, loss of balance, Hungary

Describes in general terms, unspecific expressions, Lithuania

Madness fits, India

Your illness is the consequence of addiction, Morocco

Relationship difficulties. Tendency to imagine things (...), France

I tell him he has anemia and a lack of iron, Lebanon

But without using the terms of the disease but much more

construction words, Algeria

Why do you make these incoherent speeches, pray because this is 
Because it's the disturbance of the brain, it's crazy, it refers to a degree of madness, to be disturbed, France

It's an unpleasant word. When I hear it, my soul hurts, Spain the work of the devil, Madagascar

Loss of control over oneself, misconceptions to be forgotten,

Lebanon

Having a sick head, hearing voices, Spain

Without aggression, you must eat, you must take your treatment,

Mauritania

Nervous breakdown, Italy

Often we talk about the voices in his head if they are nice or aggressive, if he always hears them. Why take pills if the voices stay in his head?, France

I describe his perceptions, behaviors and alert him if necessary, Canada

Common language: Medicines to soothe him, to cure his illness to help him move forward in his life. He has made progress. I use simple words, France

Appendix I- Quotations from verbatim for depressive episode and schizophrenia: motivations of negative feelings, rephrasing, and communication

\section{References}

1. Reed GM, First MB, Kogan CS, Hyman SE, Gureje O, Gaebel W et al (2019) Innovations and changes in the ICD-11 classification of mental, behavioural and neurodevelopmental disorders. World Psychiatry 18(1):3-19

2. Parnas J (2005) Clinical detection of schizophrenia-prone individuals. Br J Psychiatry 187(S48):s111-s112

3. World Health Organization (2008) Policies and practices for mental health in Europe. https://www.euro.who.int/pubrequest. Accessed 10 Feb 2020

4. World Health Organization (2010) User empowerment in mental health-a statement by WHO Regional Office for Europe. WHO Regional Office for Europe. https://www.euro.who.int/pubrequest . Accessed 10 Feb 2020

5. Perkins A, Ridler J, Browes D, Peryer G, Notley C, Hackmann C (2018) Experiencing mental health diagnosis: a systematic review of service user, clinician, and carer perspectives across clinical settings. Lancet Psychiatry 5(9):747-764

6. Herbay A, Bacon E (2006) Spécificité de la représentation de leur pathologie chez des patients schizophrènes ayant été informés du diagnostic. In: Le diagnostic en psychiatrie : questions éthiques. Masson, pp 45-59. https://www.cra-rhone-alpes.org/cid/opac_css/ index.php?lvl=notice_display\&id=7403. Accessed 10 Feb 2020

7. Oaks D (2012) Let's find language more inclusive than the phrase "Mentally ill"!. https://mindfreedom.org/kb/not-mentally-ill/. Accessed 10 Feb 2020

8. Pomey MP, Flora L, Karazivan P, Dumez V, Lebel P, Vanier MC et al (2015) The Montreal model: the challenges of a partnership relationship between patients and healthcare professionals. Sante Publique 27(1 Suppl):S41-S50

9. Roelandt JL, Daumerie N, Defromont L, Caria A, Bastow P, Kishore J (2014) Community mental health services: an experience from France. J Ment Heal Hum Behav 19(1):10-18

10. Reed GM (2011) Vers la CIM-11: créer un espace pour une diversité de perspectives. Inf Psychiatr 87(3):169

11. Roelandt J-L, Caria A, Defromont L, Vandeborre A, Daumerie N (2010) Représentations sociales du « fou », du « malade mental» et du « dépressif » en population générale en France. Encephale 36(3):7-13

12. Thornicroft $\mathrm{G}$ (2008) Stigma and discrimination limit access to mental health care. Epidemiol Psichiatr Soc 17(1):14-19
13. Whiteford HA, Ferrari AJ, Degenhardt L, Feigin V, Vos T (2015) The global burden of mental, neurological and substance use disorders: an analysis from the global burden of disease study 2010 . Forloni G, editor. PLoS One 10(2):e0116820

14. Thornicroft G, Chatterji S, Evans-Lacko S, Gruber M, Sampson N, Aguilar-Gaxiola S et al (2017) Undertreatment of people with major depressive disorder in 21 countries. Br J Psychiatry 210(02):119-124

15. Beck F, Guignard R (2012) La dépression en France (2005-2010): prévalence, recours au soin et sentiment d'information de la population. Sante Homme 421:43-45

16. Thornicroft G, Brohan E, Rose D, Sartorius N, Leese M (2009) Global pattern of experienced and anticipated discrimination against people with schizophrenia: a cross-sectional survey. Lancet 373(9661):408-415

17. Guillemain H (2018) Schizophrènes au XXe siècle : des effets secondaires de l'histoire. Alma 328. https://www.alma-editeur.fr/ schizophrene_au_xxe_siecle.html. Accessed 10 Feb 2020

18. McDonell MG, Short RA, Berry CM, Dyck DG (2003) Burden in schizophrenia caregivers: impact of family psychoeducation and awareness of patient suicidality. Fam Process 42(1):91-103

19. Palazzolo J (2003) Informer le patient en psychiatrie : rôle de chaque intervenant: entre légitimité et obligation. Masson

20. Uçok A, Polat A, Sartorius N, Erkoc S, Atakli C (2004) Attitudes of psychiatrists toward patients with schizophrenia. Psychiatry Clin Neurosci 58(1):89-91

21. Sato M (2006) Renaming schizophrenia: a Japanese perspective. World Psychiatry 5(1):53-55

22. Bergsholm P (2016) Is schizophrenia disappearing? The rise and fall of the diagnosis of functional psychoses: an essay. BMC Psychiatry $16(1): 387$

23. Takahashi H, Ideno T, Okubo S, Matsui H, Takemura K, Matsuura $M$ et al (2009) Impact of changing the Japanese term for "schizophrenia" for reasons of stereotypical beliefs of schizophrenia in Japanese youth. Schizophr Res 112(1-3):149-152

24. Guloksuz S, van Os J (2018) The slow death of the concept of schizophrenia and the painful birth of the psychosis spectrum. Psychol Med 48(02):229-244

25. Roelandt J-L, Askevis-Leherpeux F, Baleige A, Fontaine A, Guernut M (2018) Participation of users and carers in a reading of the international classification of diseases for mental and behavioural disorders (ICD 11) — princeps research on two disorders: depressive episode and schizophrenia. https://www.ccomssantementa 
lelillefrance.org/sites/ccoms.org/files/pdf/190225_RapportEta pe.pdf. Accessed 10 Feb 2020

26. Pelletier J (2017) Contribution of service users to the revision of WHO International Classification of Diseases: should recovery also apply to general practice? https://www.semanticscholar.org/ paper/Contribution-of-Service-Users-to-the-Revision-of-of-Pelle tier/47625f28126e7482a1ce0939aa2b7f29976e1ecf. Accessed 10 Feb 2020

27. Reinert M (1990) Alceste une méthodologie d'analyse des données textuelles et une application: Aurelia De Gerard De Nerval. Bull Sociol Methodol Méthodologie Sociol 26(1):24-54
28. Roelandt J-L, Caria A, Defromont L, Vandeborre A, Daumerie N (2010) Représentations sociales $\mathrm{du}$ « fou », du « malade mental» et du « dépressif » en population générale en France. Encephale 36(3):7-13

29. Borkman T (1976) Experiential Knowledge: a new concept for the analysis of self-help groups. Soc Serv Rev 50(3):445-456

30. Hackmann C, Balhara YPS, Clayman K, Nemec PB, Notley C, Pike K et al (2019) Perspectives on ICD-11 to understand and improve mental health diagnosis using expertise by experience (INCLUDE Study): an international qualitative study. Lancet Psychiatry $6(9): 778-785$

\title{
Affiliations
}

\begin{abstract}
Jean-Luc Roelandt ${ }^{1,2,3}$ - Antoine Baleige ${ }^{1,2} \cdot$ Marie Koenig $^{1,2} \cdot$ Vincent Demassiet $^{1,2,4}$ Mohamed Agoub $^{5}$. Victoria Barikova ${ }^{6}$. Dalila Benmessaoud ${ }^{7}$. Floriane Brunet $^{1,2} \cdot$ Mauro-Giovanni Carta $^{8}$. Giulio Castelpietra ${ }^{9}$. David Crepaz-Keay ${ }^{10}$. Nicolas Daumerie ${ }^{1,2}$. Audrey Fontaine ${ }^{1,2}$. Neringa Grigutyte ${ }^{11}$. Jugal Kishore ${ }^{12}$. Marta Kiss $^{13}$. Marc Laporta ${ }^{14}$. Elkhansaa Layoussif ${ }^{5}$. Youssouf Limane ${ }^{15}$. Marcelino Lopez ${ }^{16}$. Gioia Mura ${ }^{8}$. Jean-François Pelletier ${ }^{17} \cdot$ Mbolatiana Raharinivo $^{18}$. Sami Richa ${ }^{19} \cdot$ Rebecca Robles-Garcia $^{20}$. Anne-Claire Stona ${ }^{1,2}$. Marina Skourteli ${ }^{21}$. Catherine Thévenon ${ }^{1} \cdot$ Michel Triantafyllou $^{6} \cdot$ Fotis Vasilopoulos $^{21} \cdot$ Stéphanie Wooley $^{22}$. Geoffrey Reed $^{23} \cdot$ Mathilde Guernut $^{1,2} \cdot$ Shekhar Saxena ${ }^{23} \cdot$ Françoise Askevis-Leherpeux $^{1,2,3}$
\end{abstract}

1 EPSM Lille-Métropole, French WHO CC, Armentières, France

2 French WHO CC, Lille- Hellemes, France

3 Equipe Inserm, Eceve (UMR 1123), Paris, France

4 French Hearing Voices Network, Paris, France

5 Centre Psychiatrique Universitaire Ibn Rochd, Casablanca, Morocco

6 Centre DAccueil Et de Soins Hospitaliers, Nanterre, France

7 Etablissement Hospitalo-Universitaire Spécialisé de Psychiatrie Mahfoud BOUCEBCI, Chéraga, Algérie

8 Centro di Psichiatria e Psicosomatica Azienda Ospedaliero, Universitaria di Cagliari, Cagliari, Italia

9 Primary Care Services Area, Central Health Directorate, Regione Friuli Venezia Giulia, Trieste, Italy

10 Mental Health Foundation, London, UK

11 Vilnius University, Vilnius, Lithuania

12 Safdarjung Hospital, New Delhi, India
13 Community Psychiatry Centre of Semmelweis, Awakenings Foundation, Budapest, Hungary

14 Department of Psychiatry, McGill University, WHO CC, Montreal, Canada

15 Ministère de la Santé, Nouakchott, Mauritanie

16 Fundación Pública Andaluza para la Integración Social de Personas con Enfermedad Mental (FAISEM), Seville, Spain

17 Centre de Recherche de l'Institut Universitaire en Santé Mentale de Montréal, Montréal, Canada

18 Ministère de la Santé Publique, Tananarive, Madagascar

19 Hôpital Hôtel de Dieu de France, Beyrouth, Lebanon

20 Instituto National de Psiquatria "Ramón de la Fuente Muñiz", Mexico, Mexico

21 Association for Regional Development and Mental Health (EPAPSY), Athens, Greece

22 Mental Health Europe, Bruxelles, Belgium

23 Department of Mental Health and Substance Abuse, World Mental Health Organization, Geneva, Switzerland 